\title{
Discovery of very-high-energy $\gamma$-ray emission from a hard-X-ray bright HBL RX J1136.5+6737
}

\author{
Masaaki Hayashida* \\ Institute for Cosmic-Ray Research (ICRR), the University of Tokyo, Japan \\ E-mail: mahavadicrr.u-tokvo.ac.ip
}

\section{Hajime Takami}

High Energy Accelerator Research Organization (KEK), Japan

\section{Yusuke Konno}

Kyoto University, Japan

\section{for the MAGIC collaboration}

\section{Ryosuke Itoh}

\section{Hiroshima University, Japan}

RX J1136.5+6737 $(z=0.1342)$ is a hard X-ray bright high-peaked frequency BL Lac object as listed in the MAXI 3-year catalog as well as the Swift-BAT catalog. The source has also been detected by Fermi-LAT with a hard photon index of $1.71 \pm 0.08$ and belongs to the first Fermi-LAT catalog of $>10 \mathrm{GeV}$ sources, showing bright (photon flux $=11.7 \times 10^{-11} \mathrm{ph} \mathrm{cm}^{-2} \mathrm{~s}^{-1}$ ) emission above $10 \mathrm{GeV}$. MAGIC observed the source for about 30 hours in 2014 and discovered very high energy (VHE) $\gamma$-ray emission from the source with $>5 \sigma$ significance. The averaged flux measured by MAGIC during the 2014 observations corresponds to about $1.3 \%$ of the Crab Nebula flux at energies above $300 \mathrm{GeV}$ without significant variability. The measured spectrum shows evidence of extending into the $\mathrm{TeV}$ energy range, even though most extragalactic background light models predict the distance of $z=0.1342$ is beyond the "Cosmic $\gamma$-ray horizon" at $1 \mathrm{TeV}$. Along with the MAGIC observations, we coordinated simultaneous multi-band observations in Xray and UV bands by Swift, and in optical-IR bands by ground-based telescopes such as Kanata and KVA. In this contribution, the first results of the MAGIC discovery of VHE emission from RX J1136.5+6737 will be reported. We will also discuss origins of the $\gamma$-ray emission with a broad-band spectral energy distribution using our emission model.

The 34th International Cosmic Ray Conference,

30 July- 6 August, 2015

The Hague, The Netherlands

\footnotetext{
* Speaker.
} 


\section{Introduction}

High-frequency peaked BL Lac objects (HBLs) have the peaks of the lower energy bump in the spectral energy distribution (SED) at UV to X-ray energies (see e.g.,[[D]). Their SEDs have been well explained by synchrotron self-Compton (SSC) models in which the high energy end of the electron spectrum is responsible for both X-ray and very high energy (VHE: $>50 \mathrm{GeV}) \gamma$-ray emission (e.g., [[]]). The observed correlations of the X-ray and VHE $\gamma$-ray fluxes during large flares provide strong experimental evidence for the SSC mechanism in HBLs (e.g.,[[2]]).

VHE $\gamma$-ray photons interact with photons of the extra-galactic background light (EBL) during propagation through intergalactic space (e.g., $[[]]$ ). The produced secondary $\mathrm{e}^{+} / \mathrm{e}^{-}$subsequently generate $\gamma$ rays by Compton-scattering target photons of the EBL and the cosmic micro-wave background. The resultant secondary photons contribute to the $\gamma$-ray flux in $\mathrm{GeV}$ bands. Observed fluxes of the secondary emission at the Earth are affected by intergalactic magnetic fields (IGMFs) because the secondary $\mathrm{e}^{+} / \mathrm{e}^{-}$are deflected during the propagation in the intergalactic space. The detection of VHE $\gamma$ rays from distant sources therefore provides useful information on the formation of stars that make the EBL. Also the information of the secondary $\gamma$-ray flux can be used to constrain the strength of IGMFs (e.g.,[피]) and/or to disentangle hadronic and leptonic cascade scenarios [4]4, [23]. The distant $(z>0.1)$ HBLs are the best sources for those studies because of their hard intrinsic spectra in the VHE energy band. However, the distant TeV HBLs with a solid redshift measured by spectroscopic observations are still limited: for instance, currently we know only 12 HBLs above $z=0.13$.

In this context, the hard X-ray (a few to tens of $\mathrm{keV}$ ) flux is a useful indicator to search for new VHE HBLs with a hard photon spectrum in the (sub-)TeV band. Under the SSC scenario, the same electron energies $\left(\gamma \sim 10^{5-6}\right)$ are responsible for the hard $\mathrm{X}$-ray and $\mathrm{TeV}$ energy bands by the synchrotron emission and IC scattering in the cases for HBLs. A source with bright hard X-ray emission can also show bright VHE $\gamma$-ray emission well extending to the $\mathrm{TeV}$ energies.

Here, we utilized the X-ray information from the Monitor of All-sky X-ray Image (MAXI) onboard the International Space Station (ISS), which started scientific operations on 2009 August 15. The MAXI Gas Slit Camera (GSC) has provided unbiased X-ray samples with the best sensitivity so far in the 4-10 keV band by its all-sky survey observations. The latest MAXI-GSC catalog in the 4-10 keV band (the 2nd catalog with 37 month data [प]) includes 13 BL Lacs (all HBLs). All of them are also in the Swift-BAT 70 month hard X-ray catalog. It is interesting that 9 sources among 13 BL Lacs are already known TeV emitters and the first three sources are famous classical TeV sources, namely, Mkn 421, Mkn 501 and 1ES 1959+650. RX J1136.5+6737 is associated with 2MAXI J1136+675 whose X-ray flux in the catalog is $(14.4 \pm 0.9) \times 10^{-12} \mathrm{erg} \mathrm{cm}^{-2} \mathrm{~s}^{-1}$, and is the 7th brightest BL Lac in the $4-10 \mathrm{keV}$ band in the MAXI catalog. The source is listed both in the MAXI-GSC (4-10 keV) and in the Swift-BAT (14-195 keV) catalogs and is one of the brightest blazars in the hard X-ray band; a synchrotron component from the source is expected to be extending beyond $10 \mathrm{keV}$.

RX J1136.5+6737 is also associated with sources in the third Fermi-LAT source catalog (3FGL:[四) as 3FGL J1136.6+6736 and the first Fermi-LAT source catalog above $10 \mathrm{GeV}$ (1FHL:[[]]), with significant detections above $10 \mathrm{GeV}$ in both catalogs. A hard photon index of $1.71 \pm 0.08$ above $100 \mathrm{MeV}$ is quoted in the 3FGL catalog. 


\section{Observations and Results}

\subsection{VHE $\gamma$-ray observations with MAGIC}

MAGIC is a system of two Imaging Atmospheric Cherenkov Telescopes (IACTs) with $17 \mathrm{~m}$ dishes, located at the Canary Island La Palma ( $28^{\circ} .2 \mathrm{~N}, 17^{\circ} .8 \mathrm{~W}, 2225 \mathrm{~m}$ a.s.1.).

RX J1136.5+6737 was observed with the MAGIC telescopes on 35 nights between January 29 and May 28 in 2014 with a zenith angle range from $38^{\circ}$ to $46^{\circ}$. The observations were performed in the so-called wobble mode, where the object is observed with an $0^{\circ} .4$ offset from the camera center, and three OFF regions were used to estimate the background. The data with a total effective observation time of $28.07 \mathrm{hrs}$ after quality selections were used for the following analysis.

The analysis was performed using the MAGIC analysis package "MARS" with the standard procedure [[13]. Every cleaned event was parameterized using the so-called Hillas parameterization [प]]. These parameters were used for $\gamma /$ hadron separation by means of the "Random Forest (RF)" method [B]. In the RF method, Monte-Carlo-simulated $\gamma$-ray samples with the same zenith angle range as the data were used as $\gamma$-ray training sample while real data were used as hadronevent background sample. The $\gamma$-ray energies were reconstructed by means of energy look up tables.

Significant VHE $\gamma$-ray emission was detected with more than $5 \sigma$ excess consistent with the position of the source. Figure $\square$ shows a spatial distribution of test statistic (TS) values for the $\gamma$-ray event excess around RX J1136.5+6737. The TS value corresponds to Eq. 17 in [ए2], applied on a smoothed and modeled background estimation. Its null hypothesis distribution mostly resembles a Gaussian function, but in general can have a somewhat different shape or width.

The top panel of Figure $\square$ shows a light curve of the VHE $\gamma$-ray flux above $300 \mathrm{GeV}$ as measured with the MAGIC telescopes binned by each observing period. The observing period is defined from a full moon to the next full moon, roughly corresponding to a 28 days interval. There

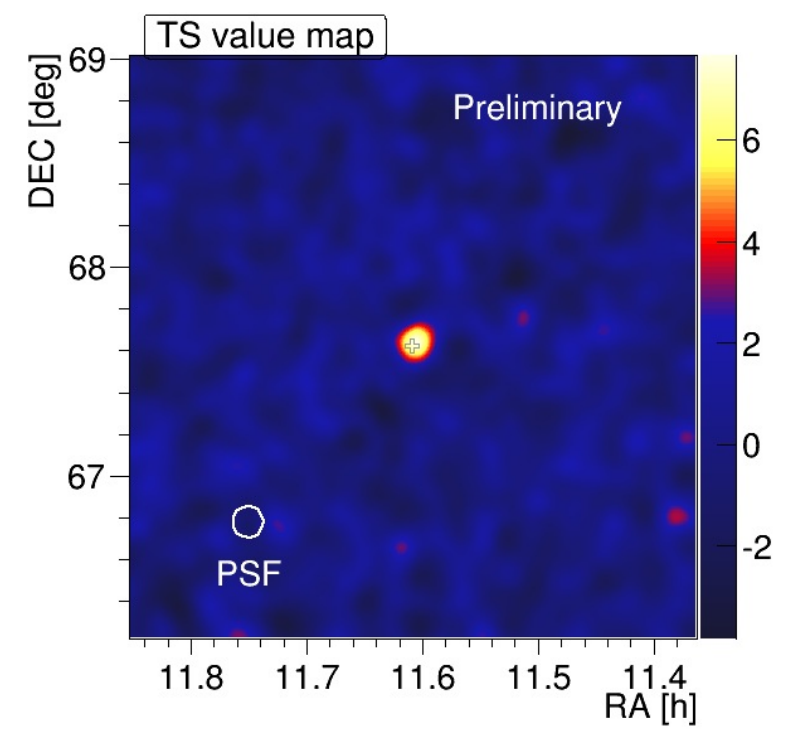

Figure 1: A sky map of the TS values of $\gamma$-ray event excess around the target, RX J1136.5+6737. The cross at the center denotes the position of the target. The excess is consistent with a point-like source. 
was no MAGIC observation in gaps between the data points because of presence of moon light. To investigate variability in the VHE $\gamma$-ray emission, the flux points in the light curve were fitted with a constant value. The fit yields $(1.38 \pm 0.39) \times 10^{-12}$ photon $\mathrm{cm}^{-2} \mathrm{~s}^{-1}$ with $\chi^{2} / \mathrm{dof}=3.69 / 4$, which indicates no significant variability. It corresponds to about $1.3 \pm 0.3 \%$ Crab flux above $300 \mathrm{GeV}$. We also checked a light curve with night-by-night time binning; not any short-time (daily scale) flaring activities were found. A VHE $\gamma$-ray spectrum could be extracted from $\sim 130 \mathrm{GeV}$ to above $1 \mathrm{TeV}$. According to current EBL models, the source is above the "Cosmic $\gamma$-ray horizon" for the given distance of $z=0.1342$ at these energies. This means that the $\gamma$-ray flux is attenuated

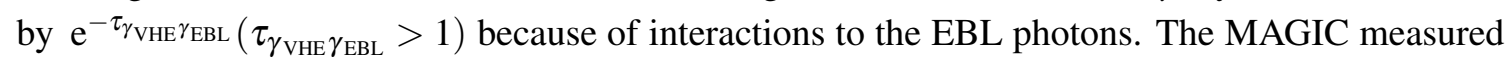
spectrum can be described by a simple power-law model with a photon index of $2.74 \pm 0.33$.

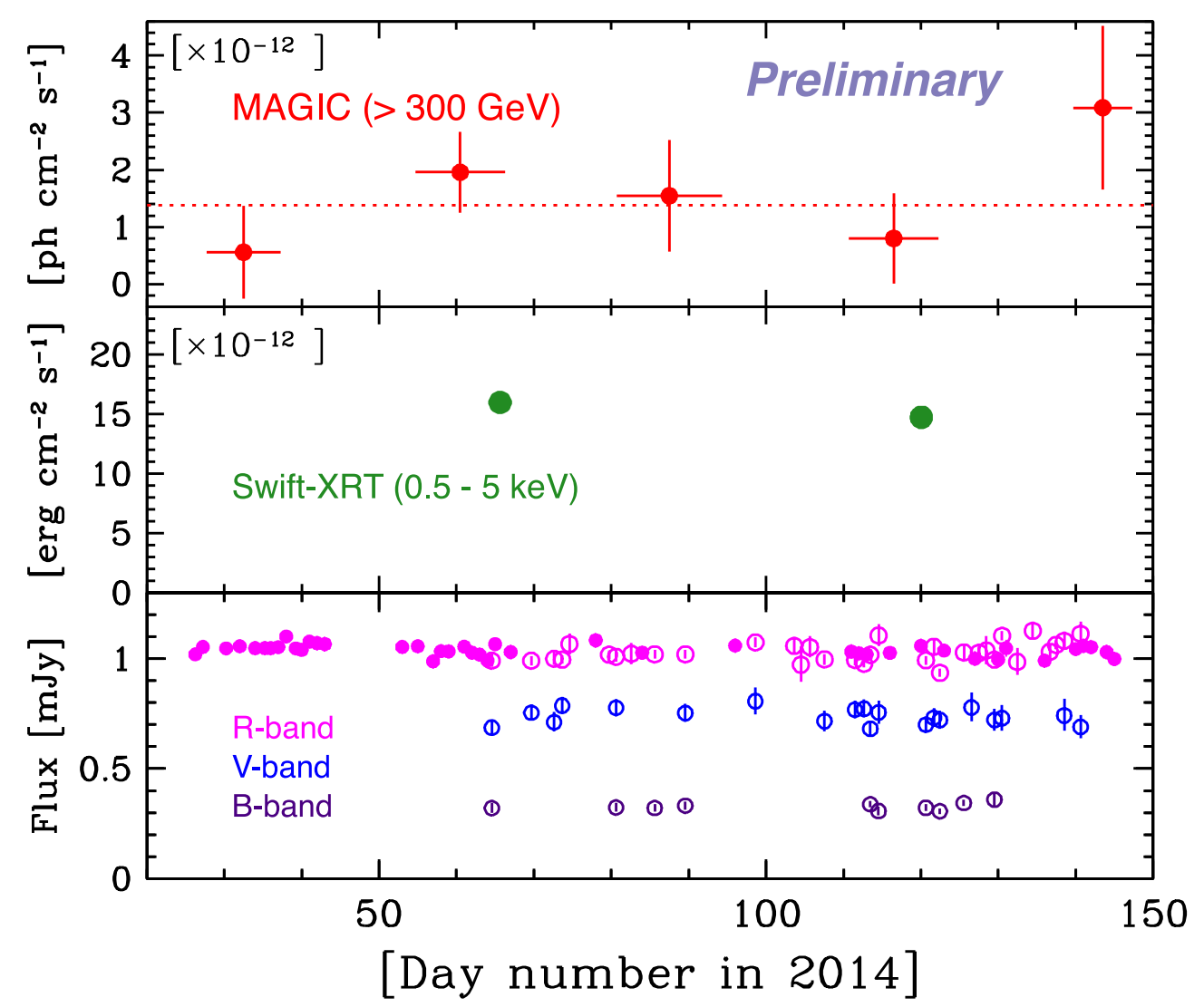

Figure 2: Light curves of RX J1136.5+6737 in several energy bands in 2014 January-May. [Top]: The VHE $\gamma$-ray band above $300 \mathrm{GeV}$ measured with the MAGIC telescopes. Each bin corresponds to one observing period, and a horizontal bar of each data point denotes a range of observing days in each period. A vertical bar at each point represents $1 \sigma$ statistical uncertainty. The horizontal dotted line corresponds to the fitting result with a constant flux to the data points. [Middle]: De-absorbed model fluxes in the $0.5-5 \mathrm{keV}$ measured by Swift-XRT. A simple power-law models is used to derive the fluxes. [Bottom]:. Optical fluxes in the $R$-, $V$ - and $B$-bands. The filled circles represent the results from KVA while the open circles are results measured with the Kanata telescope. All flux are corrected for the Galactic extinctions (de-redened fluxes). The $R$-band Katana data were artificially shifted by 0.1 magnitude brighter in the plot for a better match of the results between the telescopes. 


\subsection{Multi-band observations}

The source was observed by Swift twice during the MAGIC observing periods, on 2014 March 07 and 2014 May 01. The Swift-XRT observations were performed in Window Timing mode, and the exposure time for the observations were $1974 \mathrm{sec}$ and $1703 \mathrm{sec}$, respectively. The XRT data were reduced with the standard software xrtpipeline v.0.13.1 applying the default filtering and screening criteria. The calibration files available in the version 20140709 of the Swift-XRT CALDB were used. The spectral analysis was performed using XSPEC v.12.8.2. XRT spectra from both observations were well described by a simple power-law with a photo-electric absorption model. During the fits, the equivalent hydrogen column density was fixed to the Galactic value of $1.09 \times 10^{20} \mathrm{~cm}^{-2}$. The model fluxes between 0.5 and $5 \mathrm{keV}$ corrected for Galactic absorption in both observations are plotted in the middle panel in Figure $\square$.

In the optical-UV band, the Swift-UVOT observations used two filter bands $(V, W 1)$ on March 07 while all six filters $(V, B, U, W 1, M 2, W 2)$ were used in the observations on May 01. Source fluxes in the optical $R$-band were provided by the KVA telescope located on La Palma through their the Tuorla Observatory Blazar Monitoring Program ${ }^{1}$. The Kanata telescope located at HigashiHiroshima, Japan, conducted observations to the source in the $B$-, $V$ - and $R_{C}$-bands. The Galactic extinction in each band was derived from $E(B-V)=0.0075$ [ए8] and $A_{v} / E(B-V)=3.1$. The light curves of $R, V$ and $B$ band fluxes are plotted in the bottom panel of Figure $\square$. All fluxes were corrected for the Galactic extinctions while the host galaxy contributions are still included in the light curve fluxes.

\section{Spectral Energy Distribution}

The broad band SED of the source is shown in Figure [3. The plot includes the optical $R$ band flux measured by KVA, UV fluxes in the W2-, M1-, and W1-bands by Swift-UVOT, X-ray spectral points between $0.5 \mathrm{keV}$ and $\sim 5 \mathrm{keV}$ by Swift-XRT, high-energy $\gamma$-ray spectral points from $100 \mathrm{MeV}$ to $100 \mathrm{GeV}$ by Fermi-LAT, and the VHE $\gamma$-ray spectra obtained by MAGIC. The $R$-band flux was averaged over the MAGIC observational periods. The UV (W1, $M 2$ and $W 2$ bands) fluxes measured on 2014 May 01, and an additional W2-band flux measured on 2014 March 07 are also included. The X-ray spectrum was measured on 2014 March 07. All the fluxes in the SED plot were corrected for Galactic absorption. The host galaxy contribution estimated to be $0.85 \mathrm{mJy}$ [ए6] was already subtracted from the $R$-band flux in the SED. The $\gamma$-ray spectral points by Fermi-LAT are adapted from the 3FGL catalog. The 3FGL catalog is based on the 4 year LAT data covering only until 2012 July 13, which means that the 3FGL data range is not overlapped with the MAGIC observational periods for the source. On the other hand, the source shows no significant variability in the LAT energy band during the 4 years as indicated by the Variability index of 50.7 in $3 \mathrm{FGL}^{2}$. Furthermore, the 3FGL spectrum appears to be smoothly connected to the measured MAGIC spectrum as shown in Figure [3. Thus, the 3FGL spectrum can be assumed to represent the $\gamma$-ray spectrum in the LAT band during the MAGIC observing periods in 2014.

\footnotetext{
${ }^{1}$ More information at http://users.utu.fi/kani/1m/

${ }^{2}$ In 3FGL, Variability is considered probable when Variability Index exceeds the threshold of 72.44 corresponding to $99 \%$ confidence
} 


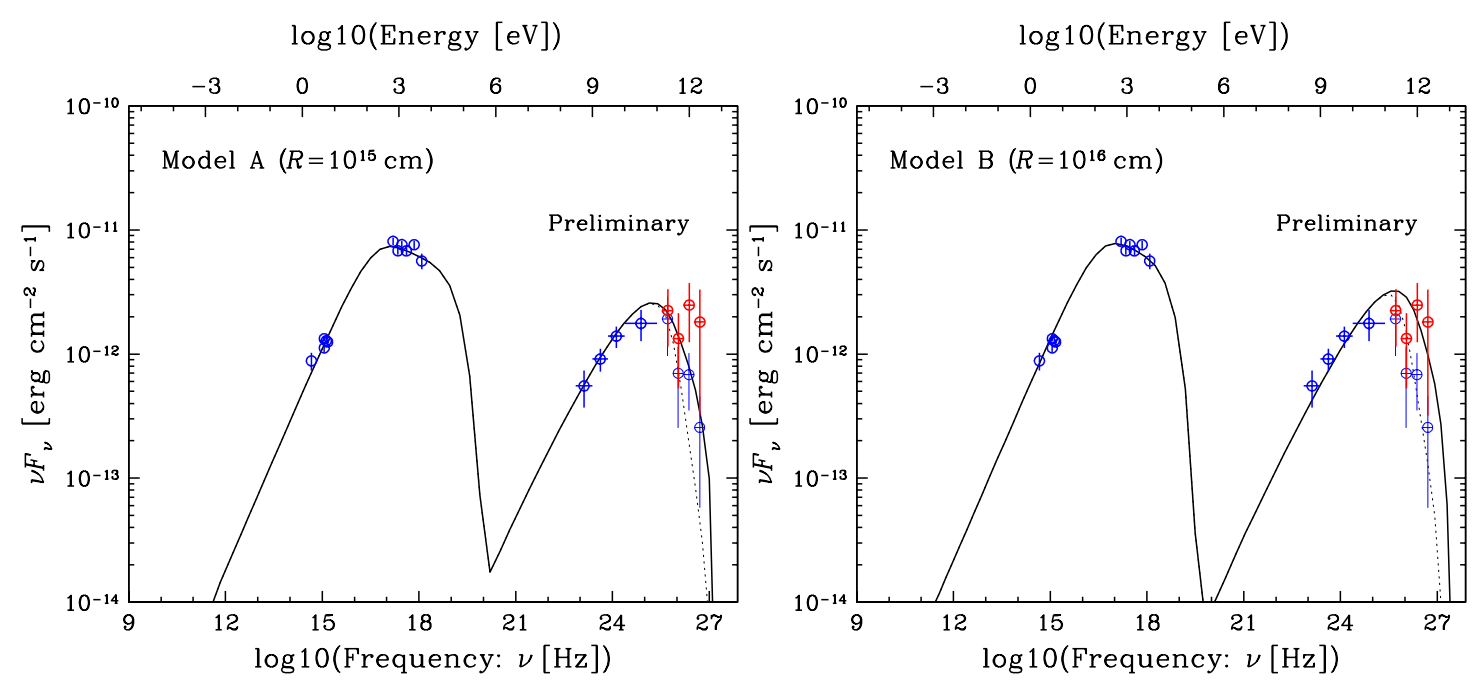

Figure 3: Spectral energy distribution of RX J1136.5+6737. The open circles represent the spectral data points. The blue circles in the VHE $\gamma$-ray band (the four highest energy points) are the measured spectral points with the MAGIC telescope while the read circles are the MAGIC data points corrected for the EBL absorption based on the model of [焑]. The data in the $\gamma$-ray bands $\left(\sim 10^{9} \mathrm{eV}\right)$ are taken from the 4-year Fermi-LAT (3FGL) catalog. The data in the X-ray bands $\left(\sim 10^{3} \mathrm{eV}\right)$ are from Swift-XRT observations on 2014 March 01. The optical $(R)$ and UV $(W 1, M 2, W 2)$ data points $\left(\sim 10^{0} \mathrm{eV}\right)$ are based on the results from KVA and Swift-UVOT observations, respectively. All X-ray and optical-UV data points were corrected for Galactic absorption. In the $R$-band data, the contribution from the host galaxy $(0.85 \mathrm{mJy})$ was subtracted. The lines represent emission modeling curves based on the one-zone SSC model for Model A (left, $R=$ $10^{15} \mathrm{~cm}$ ) and Model B (right, $R=10^{16} \mathrm{~cm}$ ). See details about the models in the text and Table 1 for the input parameters. The dotted lines in the VHE $\gamma$-ray band represent the modeling curves after attenuation due to the EBL absorption, calculated using the EBL model from [四].

\section{Emission modeling and Discussion}

A one-zone SSC model, described in [22] was applied to the broad band SED to understand the physical parameters of the jet for the emission origin. In this model, the emission region is assumed to be spherical with radius $R$ and to be filled by a tangled magnetic field of intensity $B$ in a comoving frame. The energy distribution of the injected relativistic electron is described by a broken power-law function,

$$
N(\gamma)= \begin{cases}K \gamma^{-n 1} & \left(\gamma_{\min }<\gamma<\gamma_{\mathrm{bk}}\right) \\ K \gamma_{\mathrm{bk}}^{S_{2}-s_{1}} \gamma^{-s_{2}} & \left(\gamma_{\mathrm{bk}}<\gamma<\gamma_{\max }\right),\end{cases}
$$

where $K$ is the normalization factor of the electron density, extending from $\gamma_{\min }$ to $\gamma_{\max }$ with indices $s_{1}$ and $s_{2}$ below and above $\gamma_{\mathrm{bk}}$. Relativistic effects are taken into account by the Doppler beaming factor $\delta$.

As described in Figure [1, we consider two emission models with different emission radii, namely $10^{15} \mathrm{~cm}$ (Model A: left) and $10^{16} \mathrm{~cm}$ (Model B: right). The parameters of the SSC models are summarized in Table $\mathrm{W}$. Generally speaking, the models could reproduce the observed broad band SEDs. However, if we discuss the details, the model curves in the MAGIC energy range are 
Table 1: Parameters of the SSC models presented in Figure 3.

\begin{tabular}{lll}
\hline \hline Model & A & B \\
\hline$R[\mathrm{~cm}]$ & $10^{15}$ & $10^{16}$ \\
$\delta$ & 30 & 20 \\
$B[\mathrm{G}]$ & 0.6 & 0.065 \\
$K\left[\mathrm{~cm}^{-3}\right]$ & 2600 & 130 \\
$\gamma_{\min }$ & 1 & 1 \\
$s_{1}$ & 1.8 & 1.8 \\
$\gamma_{b \mathrm{k}}$ & $3 \times 10^{4}$ & $9 \times 10^{4}$ \\
$s_{2}$ & 3.2 & 3.2 \\
$\gamma_{\max }$ & $5 \times 10^{5}$ & $1 \times 10^{6}$ \\
\hline
\end{tabular}

not as hard as the observed spectrum; Model A under-estimates the flux around $\mathrm{TeV}$ corresponding to the highest two points by MAGIC while Model B over-estimates the flux around $100 \mathrm{GeV}$ for the first two points by MAGIC. Another point is that the we have to set an electron index for the lower parts in the electron distribution as $s_{1}=1.8$ for both cases to reproduce a sharp rise in the SED from the optical-UV to the X-ray bands. Electrons with an index harder than 2 cannot be expected from the standard shock acceleration (Fermi-I) mechanisms. On the other hand, such a hard index can be generated, for example, by stochastic accelerations, which is phenomenologically equivalent to the second-order Fermi acceleration (Fermi-II) mechanism (e.g., [ए], [, 团), or by magnetic reconnection (e.g.,[Q]]). Alternatively, high values for $\gamma_{\min }$ to avoid having lower-energy electrons could also produce such a sharp rise. Those observational features, the hard photon index in the sub-TeV to $\mathrm{TeV}$ bands and the sharp rising of the synchrotron component have been observed in so-called "extreme HBLs". The extreme HBLs usually require a hard photon index of the electron spectrum and/or a high $\gamma_{\text {min }}$ for simple one-zone SSC models (e.g.,[[R4, [25]). Secondary $\gamma$-ray cascades can be another possible origin of the emission in the $\mathrm{GeV}$ (to sub-TeV) band (e.g.,[ㅍ]).

\section{Acknowledgments}

We would like to thank the Instituto de Astrofísica de Canarias for the excellent working conditions at the Observatorio del Roque de los Muchachos in La Palma. The financial support of the German BMBF and MPG, the Italian INFN and INAF, the Swiss National Fund SNF, the ERDF under the Spanish MINECO (FPA2012-39502), and the Japanese JSPS and MEXT is gratefully acknowledged. This work was also supported by the Centro de Excelencia Severo Ochoa SEV-2012-0234, CPAN CSD2007-00042, and MultiDark CSD2009-00064 projects of the Spanish Consolider-Ingenio 2010 programme, by grant 268740 of the Academy of Finland, by the Croatian Science Foundation (HrZZ) Project 09/176 and the University of Rijeka Project 13.12.1.3.02, by the DFG Collaborative Research Centers SFB823/C4 and SFB876/C3, and by the Polish MNiSzW grant 745/N-HESS-MAGIC/2010/0. 


\section{References}

[1] Acero, F., Ackermann, M., Ajello, M., et al. 2015, ApJS, in press

[2] Ackermann, M., Ajello, M., Allafort, A., et al. 2013, ApJS, 209, 34

[3] Albert, J., Aliu, E., Anderhub, H., et al. 2008, NIMA, 588, 424

[4] Asano, K., \& Hayashida, M. 2015, ApJL, 808, L18

[5] Asano, K., Takahara, F., Kusunose, M., Toma, K., \& Kakuwa, J. 2014, ApJ, 780, 64

[6] Franceschini, A., Rodighiero, G., \& Vaccari, M. 2008, A\&A, 487, 837

[7] Ghisellini, G., Celotti, A., Fossati, G., et al. 1998, MNRAS, 301, 451

[8] Gould, R. J., \& Schreder, G. 1966, Physical Review Letters, 16, 252

[9] Guo, F., Li, H., Daughton, W., \& Liu, Y.-H. 2014, Physical Review Letters, 113, 155005

[10] Hillas, A. M. 1985, Proc. 29th Int. Cosmic Ray Conf. (La Jolla), 3, 445

[11] Hiroi, K., Ueda, Y., Hayashida, M., et al. 2013, ApJS, 207, 36

[12] Li, T.-P., \& Ma, Y.-Q. 1983, ApJ, 272, 317

[13] Moralejo, A., Gaug, M., Carmona, E., et al. 2009, arXiv:0907.0943

[14] Murase, K., Dermer, C. D., Takami, H. et al. 2012, ApJ, 749, 63

[15] Neronov, A., \& Vovk, I. 2010, Science, 328, 73

[16] Nilsson, K., Pursimo, T., Heidt, J., et al. 2003, A\&A, 400, 95

[17] Padovani, P. \& Giommi, P. 1995, ApJ, 444, 567

[18] Schlafly \& Finkbeiner 2011, ApJ, 737, 103

[19] Stawarz, Ł., \& Petrosian, V. 2008, ApJ, 681, 1725

[20] Sugizaki, M., Mihara, T., Serino, M. et al. 2011, 63, 635

[21] Takahashi, T., et al. 1996, ApJL, 470, L89

[22] Takami, H. 2011, MNRAS, 413, 1845

[23] Takami, H., Murase, K. \& Dermer, C. 2013, ApJL, 771, L32

[24] Tanaka, Y. T., Stawarz, Ł., Finke, J., et al. 2014, ApJ, 787, 155

[25] Tavecchio, F., Ghisellini, G., Ghirlanda, G., et al. 2009, MNRAS, 399, L59 